\title{
Effets du type de fertilisation sur la productivité du Jatropha curcas en zone des savanes d'Afrique Centrale cas de la localité de Sanguéré Paul au Nord Cameroun
}

\author{
Adamou Issa ${ }^{1}$, Sali Bourou ${ }^{12^{*}}$, Mana Pierre Hamadou', Patrick Prudent ${ }^{182}$ et Jean LEROY1\&3 \\ 1 Institut de Recherche Agricole pour le Développement, Station polyvalente IRAD de Garoua BP 415, Cameroun \\ ${ }^{2}$ Centre de coopération internationale en recherche agronomique pour le développement Cirad-Persyst, UR Hortsys, \\ Avenue Agropolis, 34398 Montpellier Cedex 5, France \\ ${ }^{3}$ Service de la coopération et action culturelle, Ambassade de France au Cameroun ,140 av du Président Ahmadou \\ Ahidjo BP 1616, Yaoundé, Cameroun \\ *Corresponding author: sali.bourou@gmail.com
}

Original submitted in on 29th January 2016. Published online at www.m.elewa.org on 31st March 2016 http://dx.doi.org/10.4314/jab.v99i1.9

\section{RESUME}

Objectifs : L'essai a été conduit sur le site expérimental de l'IRAD station polyvalente de Garoua à Sanguéré Paul au Nord Cameroun, II a été mise en place en juillet 2009. II visait à étudier la courbe de réponse du jatropha à la fumure organique et/ou à la fumure minérale (N.P.K.) par rapport au témoin sans fertilisation. Méthodologie et Résultats : Le dispositif expérimental est du type blocks de Fischer randomisés à un facteur de 3 traitements ( 0 engrais, $200 \mathrm{~kg} / \mathrm{ha}$ de N.P.K.et $5 T$ de fumier) avec 8 répétitions. Différents paramètres ont été étudiés notamment le pourcentage de survie des plants après 5 ans, les paramètres de morphologique (hauteur, diamètre des plants, nombre de ramifications) et les paramètres de rendement dont notamment le rendement des graines par plant puis une l'évaluation séparée du rendement par hectare pour chaque traitement. Les analyses des résultats effectuées par le STATBOX et le test de Student-Newman-Keul's Multiple ( $\mathrm{P}=0.05)$ ont permis de montrer que la fertilisation a eu un effet significatif sur la hauteur des plants les deux 1 éres années et pas d'effet sur les trois dernières années. Par contre elle a eu un effet significatif sur les cinq années d'observations sur le diamètre, le nombre de ramifications, le rendement en graine par plant et à l'hectare. Toutefois l'engrais minéral s'est avéré meilleur les deux 1éres années sur la hauteur $(83,32 \mathrm{~cm}$ en 2010 et $150 \mathrm{~cm}$ en 2014), et pas de différence significative les trois années suivantes. Pourtant la fertilisation a eu un effet significatif pendant les cinq années d'observation sur le diamètre au collet, le nombre de ramification, le rendement par plant et par hectare en passant de 41,55 mm en 2010 à $101 \mathrm{~mm}$ en $2014 ; 4,77$ en 2010 à 32,25 en $2014 ; 26,66 \mathrm{~g}$ en 2010 à 648,82g en 2014 par plant et 66,16 kg/ha en 2010 à 1622 kg/ha en 2014 respectivement par rapport au témoin.

Conclusion et application des résultats : Ces résultats montrent que le Jatropha curcas considéré comme une plante de zone marginale qui ne nécessite pas la fertilisation, répond positivement à une fertilisation aussi bien organique que minérale. Cette fertilisation améliore nettement aussi bien les paramètres morphologiques que par le rendement.

Mots clés : fertilisation, Jatropha curcas, production, Nord-Cameroun 
ABSTRACT

Objectives: This work was conducted in Garoua (Sanguere Paul) multipurpose research station site in the North Cameroon. It was implemented in July 2009. The main objective was to evaluate the response curve of Jatropha to organic matter compare to mineral (NPK) supply and some of plants without any supply. Methodology and results: the disposal of the trial was Fisher randomized blocks with one factor and three treatment ( 0 supply, $200 \mathrm{~kg} / \mathrm{ha}$ of N.P.K.et $5 \mathrm{~T}$ of organic manure) with eight repetition. Different parameters were measured particularly the rate of survival of plant after five years, morphological parameters as high of plants, plants diameter and ramifications of the plants and finally the yield parameters as seeds number per plant and the yield per hectare per treatment. Data analysis were done by STATBOX and the test of Student and Newman-Keul's Multiple ( $P=0.05)$ was also used. The result showed that fertilization has a significant effect on the high of plant during the two first years and non-significant on the third year. However, fertilization has a significant effect on plants diameter, ramification number of branches and yield parameters during the five years of observations. Mineral fertilization seems to be better the two first starting years on the high of plants (83.32 cm in 2010 and $150 \mathrm{~cm}$ in 2014) and there is no significant difference during the three last years. Results showed also that fertilization has significant effects during the five years of observation on plants diameter, ramification number and yield parameter per hectare by going to $41.55 \mathrm{~mm}$ in 2010 to $101 \mathrm{~mm}$ in 2014; 4.77 in 2010 to 32.25 in 2014; 26.66g in 2010 to 648.82g in 2014 per plant and $66.16 \mathrm{~kg} / \mathrm{ha}$ in 2010 to $1622 \mathrm{~kg} / \mathrm{ha}$ in 2014 respectively compare to control plants.

Conclusion and results application: By these results, Jatropha curcas cannot be considered as marginal zone plant, which does not need fertilization. In fact, as it is presented by the results it responds perfectly as any other plant to mineral or organic fertilization. This fertilization improves morphological parameters and yield component.

Key words: fertilization, Jatropha curcas, production, North-Cameroun

\section{INTRODUCTION}

Le pourghère de son nom scientifique JatrophaCurcas est une plante oléagineuse non comestible appartenant à la famille des Euphorbiacées originaire de l'Amérique centrale. C'est une plante adaptée au climat tropicale et subtropicale formant des buissons de 4 à $6 m$ de haut où les températures diurnes moyennes sont de 20 à $30^{\circ} \mathrm{C}$ et la pluviométrie annuelle est comprise entre 300 et $600 \mathrm{~mm}$ (Henning, 2007). II résiste bien à la sécheresse grâce à ses racines pivotantes et à la forme de son tronc qui représente un réservoir d'eau, mais il ne peut survivre sur les terrains engorgés d'eau (Müench, 1986) ou sur des terrains mal drainés. En Afrique tropicale le Jatropha est cultivé comme haie vive pour délimiter les champs de cultures afin de les protéger contre la divagation des animaux (Ouédraogo, 2000). Les populations locales utilisent les feuilles, les fruits et graines dans la pharmacopée. Au Mali l'utilisation son huile pour fournir de l'énergie est fondamentale aujourd'hui pour le développement des certaines localités. Jatropha curcas pousse sur des sols dégradés, argileux, sableux, caillouteux, acides et même salins à faible teneur en nutriments sans aucune préparation préalable (Ouédraogo, 2000). En effet, le Jatropha est adapté aux sols marginaux, c'est-à-dire peu fertiles et impropres à la culture, avec une faible teneur en éléments nutritifs. Beaucoup d'auteurs s'accordent sur le fait que la fertilisation minérale et organique n'a pas sa raison d'être pour le Jatropha curcas (Müench, 1986, Ouedraogo, 2000 et Henning, 2007). Son système racinaire développé lui permet en effet de mobiliser les éléments des couches profondes du sol. Ce système racinaire, grâce à sa croissance rapide et sa forte ramification, semble être intéressant pour lutter contre l'érosion. Les expériences menées sur des sols susceptibles (sols ferrugineux tropicaux) montrent que le Pourghère peut donner une production raisonnable, à condition de lui apporter l'entretien nécessaire en phase de croissance, de maintenir la production par des intrants (azote et phosphore). Bien que la plante pousse sur les sols arides, il apparaît que pour obtenir des rendements significatifs, le Jatropha 
nécessite l'utilisation des engrais (Pirot et al., 2012). Ce pendant son rendement chute si l'apport en eau et la qualité du sol sont insuffisants. Prévoir un rendement optimal nécessite de planter le jatropha sur un sol fertile (Sanou, 2010). C'est dans cette

\section{METHODOLOGIE}

Dispositif expérimental: C'est un dispositif expérimental du type blocks de Fischer randomisés avec 8 répétitions et 3 traitements ( 0 engrais, $200 \mathrm{~kg} / \mathrm{ha}$ de N.P.K., et $5 T$ de fumier). La taille d'une parcelle optique qu'il est important de maitriser les exigences de cette culture en étudiant la courbe de réponse du Jatropha c. à la fertilisation minérale et/ou organique par rapport au témoin (sans apports minéral et organique).

élémentaire (4 lignes de 7 plants) est constituée de 4 lignes $\times 2 m \times 14 m$ soit $112 m^{2}$, pour une superficie totale de $2688 \mathrm{~m}^{2}$.

\begin{tabular}{|l|l|l|}
\hline $\mathrm{O}_{6} \mathrm{~T}_{2}$ & $\mathrm{O}_{6} \mathrm{~T}_{0}$ & $\mathrm{O}_{6} \mathrm{~T}_{1}$ \\
\hline $\mathrm{O}_{6} \mathrm{~T}_{2}$ & $\mathrm{O}_{6} \mathrm{~T}_{0}$ & $\mathrm{O}_{6} \mathrm{~T}_{1}$ \\
\hline
\end{tabular}

\begin{tabular}{|l|l|l|}
\hline $\mathrm{O}_{6} \mathrm{~T}_{0}$ & $\mathrm{O}_{6} \mathrm{~T}_{1}$ & $\mathrm{O}_{6} \mathrm{~T}_{2}$ \\
\hline
\end{tabular}

\begin{tabular}{|l|l|l|}
\hline $\mathrm{O}_{6} \mathrm{~T}_{1}$ & $\mathrm{O}_{6} \mathrm{~T}_{2}$ & $\mathrm{O}_{6} \mathrm{~T}_{0}$ \\
\hline
\end{tabular}

\begin{tabular}{|l|l|l|}
\hline $\mathrm{O}_{6} \mathrm{~T}_{0}$ & $\mathrm{O}_{6} \mathrm{~T}_{1}$ & $\mathrm{O}_{6} \mathrm{~T}_{2}$ \\
\hline
\end{tabular}

\begin{tabular}{|l|l|}
\hline $\mathrm{O}_{6} \mathrm{~T}_{1}$ & $\mathrm{O}_{6} \mathrm{~T}_{0} \mathrm{~T}_{2}$ \\
\hline
\end{tabular}

\begin{tabular}{|l|l|l|}
\hline $\mathrm{O}_{6} \mathrm{~T}_{1}$ & $\mathrm{O}_{6} \mathrm{~T}_{0}$ & $\mathrm{O}_{6} \mathrm{~T}_{2}$ \\
\hline $\mathrm{O}_{6} \mathrm{~T}_{1}$ & $\mathrm{O}_{6} \mathrm{~T}_{0}$ & $\mathrm{O}_{6} \mathrm{~T}_{2}$ \\
\hline
\end{tabular}

Conduites expérimentales : L'essai a été mise en place en juillet 2009 à l'aide des boutures de $30 \mathrm{~cm}$ (profondeur de semis : $20 \mathrm{~cm}$ ) pour une densité de 2500 plants à l'hectare ( $2 \mathrm{~m}$ entre les lignes et $2 \mathrm{~m}$ entre les plants). Mesures en cours de cycle : Différents paramètres ont été évalués notamment le pourcentage de survie des plants après 5 ans; les paramètres morphologiques: hauteur et diamètre des plants; nombre des branches naturelles et rejets et le poids des graines par plant et puis une évaluation séparée de rendement par hectare pour chaque traitement.

Analyse des données : Les données sont saisies sur EXCEL, puis analysées avec le logiciel STATBOX. La méthode de test de Student-Newman-Keul's Multiple ( $P=$ 0.05 ) est utilisée pour séparer les moyennes des traitements après l'analyse de la variance. 
Adamou et al. J. Appl. Biosci. 2016 Effets du type de fertilisation sur la productivité du Jatropha curcas en zone des savanes d'Afrique Centrale cas de la localité de Sanguéré Paul au Nord Cameroun.

RÉSULTATS ET DISCUSSIONS

Tableau 1 : La hauteur $(\mathrm{cm})$

\begin{tabular}{l|c|c|c|c|c}
\hline \multicolumn{7}{c}{ Hauteur } \\
\hline Traitements & $\mathbf{2 0 0 9 / 2 0 1 0}$ & $\mathbf{2 0 1 0 / 2 0 1 1}$ & $\mathbf{2 0 1 1 / 2 0 1 2}$ & $\mathbf{2 0 1 2 / 2 0 1 3}$ & $\mathbf{2 0 1 3 / 2 0 1 4}$ \\
\hline T1 & 83,32 & 150 & 159,92 & 168,70 & 204,17 \\
\hline T2 & 76,17 & 143,31 & 151,62 & 166,40 & 197,07 \\
\hline T0 & 65,22 & 138,98 & 151,55 & 164,65 & 195,67 \\
\hline PROB & 0,00236 & 0,04 & 0,123 & 0,724 & 0,334 \\
\hline Signification & ${ }^{* *}$ & ${ }^{* *}$ & NS & NS & NS \\
\hline CV & $11,06 \%$ & $5,42 \%$ & $5,67 \%$ & $5,95 \%$ & $5,94 \%$ \\
\hline${ }^{* *}:$ significatif ; NS : non significatif & \multicolumn{5}{l}{}
\end{tabular}

A la 1ère année, le NPK est le type de fertilisation qui améliore le mieux les paramètres de croissance. II ressort de ce résultat que de la 1ère année à la $2^{\mathrm{e}}$ année la fertilisation a eu un effet significatif sur la croissance en hauteur. Elle passe de $83,33 \mathrm{~cm}$ en 2010 à $150,92 \mathrm{~cm}$ en 2012 sur les parcelles ayant reçues l'engrais minéral NPK en deux ans, et est statistiquement différent des parcelles ayant reçues l'engrais organique $(76,17 \mathrm{~cm}$ à $143,31 \mathrm{~cm})$ par rapport au témoin $(65,22$ à $138,98 \mathrm{~cm})$. Pourtant, de 2012 à 2014 il y'a aucune différence significatif sur la hauteur de la plante entre les parcelles ayant reçues l'engrais et le témoin ceci pourrait s'expliquer par l'arrêt de croissance en hauteur de la plante. Ce qui montre que la fertilisation améliore la croissance radiale des plants. Toutefois l'application d'azote augmente significativement la hauteur des plants, le nombre des branches et le diamètre au collet (Sagar et al., 2011). Reinhard et al., 2005 ont rapporté que les variables de croissances (la hauteur, le diamètre, le nombre de ramification et le nombre de feuilles) répondent significativement à l'application de l'engrais minéral. Ce résultat obtenu est en corroborent ceux d'Ouédraogo (2000), Reinhard et
Tianasoa (2005), qui parle des possibilités d'amélioration de la croissance et du développement des plants de $J$. curcas, surtout la première année de plantation. De même Tchobsala et al., (2013) rapporte que l'application du NPK améliore la croissance en hauteur des plants de toutes les origines ou accessions de jatropha étudiés. Aussi ce résultat se rapproche de ceux de Reinhard et Tianasoa (2005) et Nicolino (2007) qui ont travaillé sur la culture de $\mathrm{J}$. curcas ainsi que ceux du Mémento de l'agronome (1998) sur les techniques agricoles rurales en Afrique. D'autres essais menés sur la station expérimentale de l'ICRISAT à Patancheru en Inde (Suhas $P$ Wani et al., 2006) ont révélé qu'un apport de $100 \mathrm{~g}$ d'urée et de $38 \mathrm{~g}$ de superphosphate par plant à la plantation a significativement amélioré les paramètres de la croissance, 21 mois après la plantation (la densité n'étant pas précisée) le nombre de branches par plant: 3,8 branches pour les témoins et 8,7 pour les plants ayant reçu l'engrais, la hauteur du plant a été augmenté de plus de $60 \mathrm{~cm}$ en moyenne pour les plants fertilisés (Pirot et al, . 2008).

Tableau 2 : Le diamètre $(\mathrm{mm})$

\begin{tabular}{|c|c|c|c|c|c|}
\hline \multicolumn{6}{|c|}{ Diamètre } \\
\hline Traitements & $2009 / 2010$ & $2010 / 2011$ & $2011 / 2012$ & 2012/2013 & $2013 / 2014$ \\
\hline T1 & 41,55 & 71,70 & 79,70 & 94,05 & 101 \\
\hline T2 & 37,87 & 67,28 & 75,10 & 85,65 & 91,52 \\
\hline TO & 34,95 & 63,69 & 72,70 & 83,27 & 87,95 \\
\hline PROB & 0,00021 & 0,021 & 0,009 & 0,008 & 0,0044 \\
\hline Signification & $* *$ & $\star *$ & ** & $* *$ & ** \\
\hline CV & $5,96 \%$ & $7,44 \%$ & $5,15 \%$ & $7,04 \%$ & $7,12 \%$ \\
\hline
\end{tabular}

L'effet des fertilisants sur le diamètre au collet des plants est hautement significatif $(p<0,001)$. L'analyse de variance montre que la fertilisation a affecté significativement la croissance notamment le diamètre au collet de la plante pendant les ces cinq années d'observation. Ainsi les meilleurs diamètres sont obtenus par les parcelles qui ont reçues l'engrais minéral plus précisément $41,55 \mathrm{~mm}$ en $2010 ; 71,70 \mathrm{~mm}$ en 2011 ; 


\section{Adamou et al. J. Appl. Biosci. 2016 Effets du type de fertilisation sur la productivité du Jatropha curcas en zone des savanes d'Afrique Centrale cas de la localité de Sanguéré Paul au Nord Cameroun.}

$79,70 \mathrm{~mm}$ en $2012 ; 94,05 \mathrm{~mm}$ en 2013 et $101 \mathrm{~mm}$ en 2014 qui sont statistiquement différent des diamètres des plants issus des parcelles où sont appliquées la poudrette notamment $37,87 \mathrm{~mm} ; \quad 67,28 \mathrm{~mm} ; \quad 75,10 \mathrm{~mm}$; $85,65 \mathrm{~mm} ; 91,52 \mathrm{~mm}$ respectivement en 2010,2011 , 2012, 2013 et 2014 comparée au témoin. Ce résultat se justifie par la contenance d'un mélange de molécules d'azote sous forme d'ions nitrates (NO3-) déjà assimilables, d'ions nitrites (NO2-) et d'ions ammonium $(\mathrm{NH} 4+)$, par rapport aux parcelles ayant reçues l'engrais organique où l'engrais organique contient généralement une grande proportion d'azote organique, c'est-à-dire des molécules d'azote liées et emprisonnées à la matière organique qui devront être dégradées par un cortège de microflore et bactéries du sol. Cette dégradation donne des ions ammoniums ( $\mathrm{NH} 4+$ ) avant d'être assimilable par la plante. (Romet, 2006). Ce résultat est en accord avec celui de Tchobsala et al., 2013 qui ont montré que l'application de l'engrais minéral NPK améliore significativement le diamètre des plants de jatropha passant de $45,10 \mathrm{~cm}$ pour le témoin à $50,75 \mathrm{~cm}$ pour le NPK. De même ce résultat se rapproche de celui de Sall (2007) sur les techniques agricoles de J. curcas.

Tableau 3 : Le nombre de ramification

\begin{tabular}{l|c|c|c|c|c}
\hline \multicolumn{6}{c}{ Ramifications } \\
\hline Traitements & $\mathbf{2 0 0 9 / 2 0 1 0}$ & $\mathbf{2 0 1 0 / 2 0 1 1}$ & $\mathbf{2 0 1 1 / 2 0 1 2}$ & $\mathbf{2 0 1 2 / 2 0 1 3}$ & $\mathbf{2 0 1 3 / 2 0 1 4}$ \\
\hline T1 & 4,77 & 20 & 21,60 & 29,55 & 32,25 \\
\hline T2 & 4,17 & 18,33 & 20,65 & 26,90 & 30,12 \\
\hline T0 & 3,57 & 13,42 & 16,85 & 21,47 & 28,75 \\
\hline PROB & 0,0082 & 0,0003 & 0,0021 & 0,0002 & 0,0012 \\
\hline Signification & $* *$ & $* *$ & $* *$ & ${ }^{* *}$ & $* *$ \\
\hline CV & $15,47 \%$ & $14,14 \%$ & $11,42 \%$ & $11,20 \%$ & $7,77 \%$ \\
\hline \multicolumn{7}{r}{$:$ significatif ; NS : non significatif }
\end{tabular}

Le résumé de l'analyse de variance présenté montre que l'application de l'engrais a eu un effet significatif sur le nombre de ramification sur les cinq années d'observations. Ainsi ce nombre est passé de 4,77 en $2010 ; 20$ en $2011 ; 21,60$ en $2012 ; 29,55$ en 2013 à 32,25 en 2014 sur les unités où sont appliquées l'engrais minéral NPK qui est meilleur que le nombre de ramification obtenu dans les parcelles ayant reçues l'engrais organique (4,17 en $2010 ; 18,33$ en $2011 ; 20,65$ en $2012 ; 26,90$ en 2013 et 30,12 en 2014 comparé au témoin (3,57 à 28,75 ramifications). Ces résultats sont similaires à ceux de Tchobsala et al. (2013) où l'application des diffèrent type de fertilisations ont montré un effet hautement significatif sur le nombre de ramifications, ce paramètre varie de 3,9 pour le témoin à 8,5 pour les parcelles qui ont reçues l'engrais minéral NPK, aussi Chatzithoedorou et al. (2004) ont montré un effet significatif de l'application de l'engrais sur la croissance notamment la hauteur, le diamètre de la plante et le nombre de ramifications par rapport au témoin après 150 jours après transplantation. Ce résultat peut s'expliquer par la disponibilité élevé des éléments nutritifs (NPK) qui stimule la croissance à la 1ère année de la transplantation.

Tableau 4 : Le rendement (g/arbre)

\begin{tabular}{|c|c|c|c|c|c|}
\hline \multicolumn{6}{|c|}{ Rendement (g/arbre) } \\
\hline Traitements & $2009 / 2010$ & 2010/2011 & $2011 / 2012$ & $2012 / 2013$ & $2013 / 2014$ \\
\hline T1 & 26,46 & 132,32 & 303,59 & 506,51 & 648,82 \\
\hline T2 & 15,54 & 77,73 & 247,85 & 385,18 & 469,51 \\
\hline TO & 5,76 & 28,81 & 226,59 & 336,95 & 364,81 \\
\hline PROB & 0,011 & 0,011 & 0,012 & 0 & 0,005 \\
\hline Signification & ** & ** & ** & ** & ** \\
\hline CV & $73,36 \%$ & $73,36 \%$ & $17,62 \%$ & $6,91 \%$ & $29,38 \%$ \\
\hline
\end{tabular}

L'analyse de variance montre que la fertilisation a eu impact sur les cinq années de production, toutefois le rendement obtenu par les plants ayant reçu l'engrais minéral (NPK) est statistiquement différent des parcelles 


\section{Adamou et al. J. Appl. Biosci. 2016 Effets du type de fertilisation sur la productivité du Jatropha curcas en zone des savanes d'Afrique Centrale cas de la localité de Sanguéré Paul au Nord Cameroun.}

ayant reçues la poudrette, elle aussi différent du témoin. Toutefois la production est passée de $26,46 \mathrm{~g}$ en 2010 ; $132,32 \mathrm{~g}$ en $2011 ; 303,59$ en $2012 ; 506,51 \mathrm{~g}$ en 2013 et à 648,82 g en 2014 par plant en cinq ans pour les unités expérimentales qui ont reçues l'engrais chimique est statistiquement supérieure aux parcelles à poudrettes où le rendement est passé de 15,54 g à 469,51 g par plant comparée au témoin $(5,76 \mathrm{~g}$ en $2010 ; 28,81 \mathrm{~g}$ en 2011 ; $226,59 \mathrm{~g}$ en 2013 et $364,81 \mathrm{~g}$ en 2014). Ce pendant la production est passé de 364,81 g/plant pour la parcelle témoin à 648,82 g/plant sur la parcelle ayant reçues l'engrais minéral NPK, presque le double du témoin en 2014.

Tableau 5 : Le rendement $(\mathrm{kg} / \mathrm{ha})$

\begin{tabular}{l|c|c|c|c|c}
\hline \multicolumn{7}{|c}{ Rendement (kg/ha) } \\
\hline Traitements & $\mathbf{2 0 0 9 / 2 0 1 0}$ & $\mathbf{2 0 1 0 / 2 0 1 1}$ & $\mathbf{2 0 1 1 / 2 0 1 2}$ & $\mathbf{2 0 1 2 / 2 0 1 3}$ & $\mathbf{2 0 1 3 / 2 0 1 4}$ \\
\hline T1 & 66,16 & 330,81 & 758,994 & 1266,28 & 1622,06 \\
\hline T2 & 38,86 & 194,34 & 619,64 & 962,97 & 1173,79 \\
\hline T0 & 14,40 & 72,03 & 566,47 & 842,387 & 912,03 \\
\hline PROB & 0,011 & 0,011 & 0,012 & 0 & 0,005 \\
\hline Signification & $\star *$ & $* *$ & $* *$ & ${ }^{* *}$ & ${ }^{* *}$ \\
\hline CV & $73,36 \%$ & $73,36 \%$ & $17,62 \%$ & $6,91 \%$ & $29,38 \%$ \\
\hline
\end{tabular}

$S$ : significatif ; NS : non significatif

L'étude montre un effet significatif de la fertilisation sur le rendement de jatropha à l'hectare. II en découle de ces travaux, que l'engrais minéral a entrainé une augmentation significative par rapport au témoin sur les cinq années de production. Ainsi l'application de l'engrais minéral NPK à raison de $200 \mathrm{~kg} / \mathrm{ha}$ a obtenu un meilleur rendement et est statistiquement différent de la parcelle à poudrette (5t/ha) elle-même différent du témoin. Toutefois on note une évolution de la production de la 1 ère à la $5 \mathrm{e}$ année, elle est passée de 66,16 kg/ha en 2010 à 1622,06 $\mathrm{kg} / \mathrm{ha}$ en 2014 sur les parcelles qui ont reçues l'engrais chimique NPK, et est supérieure aux traitements où est appliquée la poudrette à parc à bétail avec 38,86 en 2010 et $1173,79 \mathrm{~kg} / \mathrm{ha}$ en 2014 comparée au témoin $(14,40 \mathrm{~kg}$ en 2010 à 912,03 kg en 2014). Pirot et al.(2008) considère qu'on pourrait atteindre un rendement moyen de 2 t/ha avec des apports d'engrais, d'eau et une conduite de culture adaptée ce qui est proche de nos résultats. Plusieurs auteurs supposent qu'une ramification précoce de la plante aurait un effet déterminant sur le rendement car les inflorescences se développement seulement au bout des branches, donc plus la plante a de branches et plus elle produirait de fruits (Pirot et al., 2008). Ce résultat est similaire à celui de certains auteurs où l'engrais minéral NPK améliore la production des plants (Tchobsala et al., 2013 ) et le nombre de ramification dépend de l'adaptation de la plante et les conditions écologiques (Ouédraogo, 2000). Des résultats similaires sont obtenus où la production est passée à plus de $300 \mathrm{~g}$ par plant (Reinhard et al., 2005). Une étude menée en Inde sur la fertilisation minérale (Patolia et al., 2007) montre qu'elle contribue efficacement à l'augmentation du rendement. Ounchittikoun (2005) indique que l'application de l'engrais minéral NPK (15-1515) améliore le rendement du jatropha pendant les trois 1 éres années de production (725, $56 \mathrm{~kg} / \mathrm{ha})$. Au Mali, les rendements de l'ordre de $500 \mathrm{~kg} / \mathrm{ha}$ dans une plantation de 3 ans ont été signalés dans une agriculture à faibles niveaux d'intrants (De Jongh et Nielsen, 2011).

Tableau 6 : Taux de mortalité

\begin{tabular}{l|l}
\hline Traitement & Taux de mortalité des plants (\%) \\
\hline T1 & 0,85 \\
\hline T2 & 2,25 \\
\hline T0 & 2,87 \\
\hline PROB & 0,07 \\
\hline Signification & NS \\
\hline CV & $82,29 \%$ \\
\hline NS : non significatif &
\end{tabular}




\section{Adamou et al. J. Appl. Biosci. 2016 Effets du type de fertilisation sur la productivité du Jatropha curcas en zone des savanes d'Afrique Centrale cas de la localité de Sanguéré Paul au Nord Cameroun.}

Après cinq années d'observations l'analyse statistique montre qu'il y a aucune différence significative entre les traitements concernant le nombre de plants perdus, ainsi l'application de l'engrais n'a pas eu d'effet sur le taux de

\section{CONCLUSION}

Ce travail mené sur la réponse du Jatropha curcas en fonction du type de fertilisation a montré une grande variation sur les caractères étudiés en cinq ans d'observation. Les parcelles qui ont reçues l'engrais minéral NPK ont produits des résultats meilleurs sur la hauteur (83,32 à 204,17 cm), le diamètre au collet $(41,55$ à $101 \mathrm{~mm})$, le nombre de ramifications $(4,17$ à 32,25$)$, le rendement $(66,16$ à $1622,06 \mathrm{~kg} / \mathrm{ha})$ et sont statistiquement différent des parcelles fertilisées à l'engrais organique notamment la hauteur $(76,17$ à

\section{REMERCIEMENT}

Les auteurs de manière unanime salut l'âme de l'auteur Mana Pierre Hamadou décédé en 2011. Cet article lui

\section{BIBLIOGRAPHIE}

Chatzitheodorou, I. T., Sotiropoulos, T. E., \& Mouhtaridou, G. T. 2004. Effect of Nitrogen, Phosphorus, Potassium Fertilization and Manure on Fruit Yield and Fruit Quality of the Peach Cultivars Spring Time and Red Haven. Agronomy Research, 2(2), 135-143.

De Jongh J, Nielsen F. 2011. Lessons learned: Jatropha for local development. Fact foundation, Available from: www.fact-foundation.com

Dieye P. N. 2007. Les biocarburants: Une nouvelle donne pour les politiques agricoles. (4) 14-15 pp.

Domergue M. et Pirot R., 2008. Rapport de synthèse bibliographie sur le J. curcas. $118 \mathrm{p}$

Henning, R., 2007. Identification, selection and multiplication of high yielding J. curcas L. plants and economic key points for viable J. curcas oil production costs (Henning paper)

Mohapatr S. A, Panda P. K. 2011. Effects of Fertilizer Application on Growth and Yield of Jatropha curcas L. in an AericTropaquept of Eastern India. Not SciBiol, 2011, 3(1):95-109 p.

Munch E. et Kieffer J. 1986. Le Pourghère (Jatropha curcas L.) Botanique, écologie, culture (1ère partie), produits de récolte, filières de valorisations, réflexions économique (2ème partie), Université de Stuttgart - Hohenheim, Allemagne. 276 p. mortalité des plants. Néanmoins numériquement on note que les parcelles ayant reçues l'engrais minéral ont un faible taux de mortalité $(0,8 \%)$ suivie des unités à engrais organique $(2,25 \%)$ par rapport au témoin $(2,87 \%)$.

$197,07 \mathrm{~cm})$, le diamètre au collet (37,8 à 91,52 mm), le nombre de ramifications $(4,17$ à 30,12$)$ et le rendement à l'hectare (38,86 à 1173,79 kg/ha) par rapport au témoin. Toutefois il est important de poursuivre cette étude pour atteindre la production maximale de cette culture, et aussi de faire une étude semblable mais sur un essai mise en place par transplantation des plants issus de la pépinière, et utiliser les doses fragmentées de l'engrais minéral NPK afin de savoir la dose raisonnable pour une production optimale.

est dédié pour sa contribution majeure à ce travail. Que son âme repose en paix.

Nicolino F. 2007. La faim, la bagnole, le blé et nous : Une dénonciation des biocarburants. $175 \mathrm{p}$.

Ouédraogo M. 2000. Étude biologique et physiologique du Pourghère (Jatropha curcas L.). Thèse d'État, UO/BF. 290 p.

Ounchittikoun T. 2005. Physic nut-the energy crop for various utilizations. Bangkok: $35 \mathrm{p}$.

Patolia J.S., Ghosh A., JitendraChikara, Chaudhary D.R., Parmar D. R. and Bhuva H. M. 2005. Response of Jatropha curcas grown on wasteland to $\mathrm{N}$ and $P$ Fertilization

Pirot R. et Hamel O. 2012. Les réalités du Jatropha curcas confrontées aux opportunités des mécanismes financiers liés au carbone (Fantasme, aubaine ou réelle opportunité ?). Crédits carbone pour l'Agriculture, la Sylviculture, la Conservation et l'Action contre la Déforestation. $32 p$.

Reinhard K. H. et Tianasoa R. 2005. Le manuel Jatropha : un guide pour l'exploitation intégrée de la plante Jatropha à Madagascar. Allemagne, Green Island Association. $20 \mathrm{p}$.

Romet L. 2006. Groupe de Recherche en Agriculture Biologique. Journées Techniques Fruits \& Légumes et Viticulture Biologiques GRAB / ITAB 200613 et 14 décembre 2006 - Moissac.

Sall H.M. 2007. Rapport annuel de projet Biocarburant 2007/2012 : Nouvelle orientation de la politique 
agricole au Sénégal, Ministère de développement rural et de l'Agriculture $24 \mathrm{p}$.

Sanou F. 2010. Productivité de Jatropha curcas L. et impact de la plante sur les propriétés chimiques du sol : cas de Bagré (Centre-est du Burkina Faso). Mémoire de fin de cycle, U.P.B., I.D.R. $72 \mathrm{p}$.

Soukvilay V. 2010. Effects of different spacing and fertilization on growth and seed yield of the physic nut (Jatropha curcas L.) in the first year of plantation. A thesis submitted in partial fulfillment of the requirement of the degree of Master of Science program in agricultural resource and environmental management UdonThaniRajabhat University. $150 \mathrm{p}$.

Tchobsala, Mégueni C., Njintang Y. N., Nenwôla K. B., Prudent P., Wey J., Lyanaz J. and Djonbada P. 2013. Influence of Agricultural Inputs on Growth and Yield of Jatropha curcas (L.) in Cameroon. Journal of Life Sciences, 179134- 1934 pp. 